\title{
The effects of exposure duration and surrounding frames on direct and indirect tilt aftereffects and illusions
}

\author{
PETER WENDEROTH and RICK VAN DER ZWAN \\ University of Sydney, Sydney, New South Wales, Australia
}

\begin{abstract}
Direct and indirect tilt illusions (TIs) have been shown to have different mechanisms, because spatial parameters that affect the one do not affect the other, and vice versa. The indirect TI, for example, is reduced markedly by a surrounding vertical square frame, a manipulandum that has no effect on the direct TI (Wenderoth \& Johnstone, 1988a). In six experiments, we show that both direct and indirect TIs are enhanced in magnitude with short (10-60 msec) exposures; that tilt aftereffects (TAEs) induced with short test exposures are entirely comparable in magnitude; that a surrounding square frame reduces indirect TAEs but not direct TAEs, just as occurs with the TI; and that when the surrounding frame is present during adaptation only, test only, and both or neither, the greatest indirect TAE reduction occurs when the frame is present during the test. These results are consistent with the view (Wenderoth \& Johnstone, 1987, 1988a, 1988b) that indirect TIs and TAEs may not reflect temporary neural modification based on V1 lateral inhibitory processes but rather the operation of more global, possibly extrastriate, orientationconstancy mechanisms.
\end{abstract}

Recent experiments have shown that both the tilt aftereffect and tilt illusion (defined below) show marked increases in magnitude as the duration of the test stimulus decreases (Calvert \& Harris, 1988; Harris \& Calvert, 1989; Wolfe, 1984). It has been suggested that different processes underlie the short- and long-range effects (Wolfe, 1984), and that these processes might reflect the operation of transient and sustained subsystems in human vision (Harris \& Calvert, 1989). Other data have suggested that separate processes underlie so-called direct and indirect tilt illusions, and that the latter are produced by a higher, more sustained visual mechanism than the former (Wenderoth \& Johnstone, 1988a, 1988b). In this paper, temporal and spatial variables known to affect direct and indirect tilt illusions differentially are used to determine whether they affect tilt aftereffects in similar fashion.

If an observer adapts for some minutes to a line or grating tilted $10^{\circ}-15^{\circ}$ from vertical, a subsequently presented vertical line or grating will appear tilted in the opposite direction (a repulsion effect). Hence, in order to make this truly vertical stimulus look vertical, the observer must set it slightly in the same direction as the adapting tilt. When Gibson and Radner (1937) first measured this tilt

\footnotetext{
This research was supported by the Australian Research Grants Scheme, Grant A78515620, and Australian Research Council Grant A78831871 to the first author, which provided the second author's research assistant position. Invaluable assistance with all aspects of computing facilities was provided by John Holden. Part of this paper was presented at the 24th International Congress of Psychology, Sydney, Australia, 1988, and will be published in the Proceedings of the Congress (Vol. 6). Correspondence may be addressed to Peter Wenderoth, Department of Psychology, University of Sydney, Sydney, NSW 2006, Australia.
}

aftereffect (TAE) as a function of the degree of tilt of the adapting stimulus, they drew attention to two distinct effects. For smaller tilts of the adapting stimulus, within the range of approximately $0^{\circ}-50^{\circ}$ from vertical, the TAE was as described above: compared with pretest control settings, postadaptation settings were in the direction of the adapting stimulus with peak magnitude $1^{\circ}-2^{\circ}$ around $15^{\circ}$ adapting tilt. For larger adapting tilts, however, between about $50^{\circ}$ and $90^{\circ}$, TAEs were in the opposite direction, with peak magnitude about $0.5^{\circ}$ at approximately $75^{\circ}$ inducing tilt: the truly vertical test stimulus appeared tilted in the same direction as the adapting stimulus (an attraction effect) and so had to be set in the opposite direction in order to appear vertical. Because the former repulsion effects were due to the effect of a near-vertical adapting stimulus on a vertical test stimulus, Gibson and Radner referred to them as direct effects; and the smaller attraction effects due to near-horizontal adapting stimuli on the vertical test stimulus were termed indirect effects. Traditionally, as here, direct effects are called positive and indirect effects negative.

Subsequent research has not only confirmed the occurrence of both direct and indirect TAEs (e.g., Mitchell \& Muir, 1976; Morant \& Harris, 1965; Muir \& Over, 1970), but also shown that both of these effects occur when the inducing and test stimuli are presented simultaneously, in the paradigm termed the tilt illusion, or TI (e.g., O'Toole \& Wenderoth, 1977; Over, Broerse, \& Crassini, 1972; Wenderoth \& Johnstone, 1988a). Partly for this reason, but for others too, many authors have suggested common mechanisms for the TAE and the TI (e.g., Magnussen \& Kurtenbach, 1980; Wenderoth \& Johnstone, 1987). 
One of the most generally accepted theories of the TAE and the TI is that of Carpenter and Blakemore (1973), which asserts that the effects are due to lateral inhibitory interactions between populations of orientation-selective neurons in V1 visual cortex. According to this view, a single line or grating in the visual field will excite cells tuned to its orientation maximally, will excite cells tuned to nearby orientations but less so depending upon the proximity of their orientation preference, and will inhibit cells tuned to more remote orientations. The single line or grating will be perceived in its correct orientation because this is signaled by the most active cells, which are those tuned to the stimulus's orientation. If, however, two lines or gratings are simultaneously present-say, one vertical and one tilted $10^{\circ}-15^{\circ}$ - the inhibitory flank set up by each stimulus will subtract from the excitatory peak of the other, causing the net pair of excitatory peaks to shift apart (or toward each other, if the angular separation is smaller; see O'Toole \& Wenderoth, 1977). Since the perceived orientations depend on the most active neurons, the stimuli will repel each other perceptually, accounting for repulsion, or direct, TIs.

Direct TAEs are then simply explained by the proposition that after adaptation, the inhibition is tonic and therefore affects the test stimulus despite the physical removal of the adapting stimulus. This inhibition theory can be regarded as a contemporary version of Köhler and Wallach's (1944) satiation theory, in which perceived contours were held to induce electrical currents that caused a buildup of resistance, with subsequently presented contours being repelled from the resistance sites.

The problem for these theories, old and new, is to explain indirect effects. Inhibition and satiation are repulsion mechanisms and seem unable to account easily for remote attraction effects. Although O'Toole and Wenderoth (1977) suggested a model in which disinhibition could explain indirect effects, this has not been tested directly. Wenderoth, O'Connor, and Johnson (1986) measured TIs using linelength variations, which, according to neurophysiological evidence, should have maximized the opportunity for indirect effects to occur, if their mechanism was disinhibition. However, indirect effects were not obtained.

There is now convincing evidence that direct and indirect effects have different mechanisms, so that any complete theory of these tilt effects cannot be couched in terms of a single mechanism. In this paper, first some recently published evidence on TIs is reviewed; then some new data on TIs and TAEs are presented; finally, a theory to explain the effects is discussed, and some suggestions for future experiments are made.

\section{Spatial Modulation of Direct and Indirect TIs}

Different kinds of stimulus displays traditionally have been used to study the TI and TAE: a single, short-arm acute angle (e.g., Blakemore, Carpenter, \& Georgeson, 1970; Carpenter \& Blakemore, 1973; Köhler \& Wallach, 1944; Lennie, 1972; Virsu \& Taskinen, 1975; Wenderoth \& Johnson, 1985; Wenderoth et al., 1986); a central, cir- cular test grating surrounded by an annulus inducing grating (e.g., Georgeson, 1973; Tolhurst \& Thompson, 1975; Wenderoth \& Johnstone, 1988a); an inducing grating with a single test line (e.g., Gibson \& Radner, 1937); and relatively long inducing and test lines that intersect at their centers (e.g., Muir \& Over, 1970). Indirect effects have not been obtained with the first of these paradigms but do occur reliably with the others.

Wenderoth and Johnstone (1988a) have used the second display, a central test grating surrounded by an annulus. They note that direct TIs and closely related Zöllner illusions are reduced markedly when inducing and test components do not abut (Tolhurst \& Thompson, 1975; Virsu \& Taskinen, 1975; Wallace, 1969) or when they differ in spatial frequency (Georgeson, 1973; Ware \& Mitchell, 1974). These results are consistent with the view that direct effects arise from V1 lateral inhibition, because V1 neurons frequently are tightly tuned to spatial position and frequency. Thus, introducing gaps or frequency differences between inducing and test stimuli would reduce the overlap between induced and tested neurons, thereby reducing the TI or TAE. Wenderoth and Johnstone (1988a) asked whether these manipulations would similarly reduce indirect TIs. In Experiment 1, the width of the annulus gap between test and inducing stimulus was varied, and inducing and test gratings had the same frequency. Whereas increasing gap size caused direct TIs to fall linearly from $2.2^{\circ}$ to $1.1^{\circ}$, indirect effects were all of the order of $-0.7^{\circ}$ and were unaffected by gap size. In Experiment 2, both gap size and inducing spatial frequency were varied. Direct effects were large only under abutting/same-frequency conditions. When the gap, the frequency difference, or both occurred, direct effects decreased. Indirect effects were not systematically affected by either manipulation. In Experiment 3, the thickness of the now always abutting inducing annulus was varied. As thickness increased, direct TIs increased markedly at first, and then more slowly, showing a linear and quadratic trend. Indirect effects, on the other hand, showed no significant trend at all.

Although it can be imprudent to speculate about neuroanatomical bases from purely psychophysical data, these results seem to lead naturally to speculation that whereas direct effects might arise largely in V1, indirect effects could arise later in extrastriate cortex, for the following reasons: A number of authors (e.g., Allman, Miezin, \& McGuinness, 1985; Maunsell \& Newsome, 1987) have drawn attention to the fact that both striate and extrastriate neurons can exhibit not only a classic receptive field (CRF), but also a total receptive field (TRF). The CRF is defined as the retinal area within which the cell's response can be elicited with an appropriate stimulus; the TRF includes much more remote or distal areas, which do not influence the cell when they alone are stimulated but do modulate the cell's response to its best stimulus in the CRF. In addition to the TRF, which could be due to feedback from higher extrastriate cells to lower cells, it has also been reported that extrastriate cells often show 
little spatial selectivity. Thus, Peterhans and von der Heydt $(1987$, p. P4) have reported that "activity in V2 is more related to object concepts and less to local stimulus features such as luminance and connectedness"; and Desimone, Moran, Schein, and Ungerleider (1985) have suggested that cells in V4 and inferotemporal cortex (IT) might be concerned with global processing and constancy: they note that "the sensitivity of most IT neurons to shape appears to be based on a global property of the shape rather than on the size or location of local contours"' (p. 449). Since indirect TIs seem unaffected by gaps or annulus thickness, an extrastriate locus where global processing is the rule seems possible.

Finally, we recall that Gibson and Radner (1937) obtained their direct and indirect TAEs with no vertical or horizontal reference lines visible. Köhler and Wallach (1944) claimed that indirect effects, but not direct effects, disappeared when truly vertical or horizontal edges were present. Could this reflect broad spatial influences of the kind reported with TRFs or extrastriate receptive fields? To establish whether the same phenomenon occurred with the $\mathrm{TI}$, Wenderoth and Johnstone (1988a) surrounded the $1.5^{\circ}$-diam test grating with a $1^{\circ}$-thick inducing annulus. This whole display could be centrally embedded in a light vertical, or near-vertical square, $4.5^{\circ}$ on a side, on a darker background. The presence of the square had no effect at all on direct effects; but it eradicated, or markedly reduced indirect effects, from $-0.79^{\circ}$ to $-0.20^{\circ}$, a reduction of $75 \%$.

Wenderoth and Johnstone (1988a) concluded that direct and indirect TIs have different determinants, and they speculated regarding an extrastriate locus of the indirect effect. In addition, since gaps, frequency differences, and annulus thickness all reduced but did not eliminate direct effects, it was suggested that a small proportion of the direct effect might also arise at a higher level.

\section{Temporal Modulation of Direct and Indirect TIs}

Wolfe (1984) reported that direct TAEs increased dramatically in magnitude when test stimuli were very short flashes. Similar results were found with the direct TI by Calvert and Harris (1988), who simultaneously reduced both the test and inducing durations. It is not clear why short-duration direct effects are large. Wolfe (1984) suggested that there are two processes involved: Either there is a short process, which is highly adapted by brief stimuli, and a long process, which is highly adapted by long stimuli, or there are two processes with different adaptabilities and different latencies. The main evidence for this proposal was that a short test flash following a long one still produced a larger TAE, which refuted the argument that a longer test flash merely causes counteradaptation. However, some doubt remains, because it is possible that recovery from counteradaptation is rapid; and Wolfe was unable to obtain consistent judgments when the interstimulus interval between test flashes was less than $200 \mathrm{msec}$, so that a single mechanism remains a possibility. Harris and Calvert (1989) have found that larger TAEs occurred with low test spatial frequencies at 100 -msec test duration but that larger TAEs occurred with high spatial frequencies at 1,000 -msec test durations-a result they took to support Wolfe's (1984) two-process hypothesis and to relate it to the known properties of transient and sustained mechanisms. They accounted for Wolfe's two-flash result by suggesting that the first, long flash adapts the sustained mechanism, leaving the transient mechanism unadapted. However, in their second experiment, the interaction between spatial frequency and test-flash duration was not obtained. Thus, the question of two processes underlying short- and long-duration TAEs remains somewhat open.

Nevertheless, whatever the explanation, we have sought to use the magnification of the TI and TAE at short durations to answer the following questions:

1. Will both direct and indirect TIs increase as exposure duration decreases?

2. Will both direct and indirect TAEs increase in similar fashion?

3. Will the short-duration TIs and TAEs have similar magnitude when measured on the same apparatus?

4. Will surrounding square stimuli reduce or eradicate both indirect TIs and TAEs at short durations?

5. If so, does the surrounding square have its effect at input or output; that is, in the TAE paradigm, will indirect effects be reduced by a square frame present only during adaptation, only during test, or both?

Question 1 bears on the mechanisms of direct and indirect effects: a failure of the indirect TI to increase as the direct effect would not only add another difference to the determinants of the phenomena, but also add to the evidence that the indirect effect reflects the operation of a more sustained mechanism. Questions 2 and 3 relate to the proposal that the TIs and TAEs share common mechanisms. Of course, the mere fact that TIs and TAEs covary with some independent variable in similar fashion would not in itself imply anything about common mechanisms; but given other, independent evidence for common mechanisms (see Harris \& Calvert, 1989), it would be expected that they would behave similarly. Question 4 bears on the nature of the frame effect: the previous report of reduction in the indirect TI (Wenderoth \& Johnstone, 1988a) was based on an adjustment experiment in which the frame was clearly and cognitively present. Could the frame exert the same effect when briefly flashed with no opportunity for conscious comparisons? Question 5, which relates to the mechanism of the frame's influence, cannot be asked within the simultaneous TI paradigm but only within the TAE paradigm, in which inducing and test stimuli are presented successively: Does the frame reduce the adapting effect of the inducing grating; or does it reduce the error in judging the test stimulus?

The first question, and part of the fourth, have already been answered to some extent (Wenderoth \& Johnstone, 1988b). In a series of experiments involving flash durations between 25 and $1,600 \mathrm{msec}$, it was found that both direct and indirect TIs were larger at short durations. However, although the direct TI seemed to decrease 
monotonically with duration, the trend was less clear for the indirect TI, with some suggestion of a decrease at the shorter durations. Wenderoth and Johnstone (1988b) took this to be consistent with an extrastriate locus for the indirect effect, with the longer latency of the maximum reflecting later extrastriate processing. Wenderoth and Johnstone also tested the effect of introducing the surrounding frame and found that at all four flash durations $(25,100,400$, and $1,600 \mathrm{msec})$, the direct effect was unaffected but the indirect effect was reduced by 0.67 . Again, these data suggested smaller indirect effects at the shortest $(25-\mathrm{msec})$ duration. Let us turn now to the additional questions.

\section{EXPERIMENTS 1 AND 2}

Experiments 1 and 2 were designed as preliminary experiments to measure direct and indirect TIs at durations; shorter than the minimum $25 \mathrm{msec}$ in previous experiments. The aim was to attempt to establish whether direct TIs continued to increase at shorter durations, and whether indirect TIs did indeed become smaller again at the shortest durations.

\section{Method}

Apparatus. Stimulus displays were presented on the flat screen of a Tektronix 608 display monitor (P31 phosphor) interfaced with an Innisfree ("Picasso") Image Generator and a PDP-11/23 minicomputer. The subjects used the outer pair of three microswitches to indicate whether the test grating appeared tilted left or right of vertical. The display was viewed in a dark, windowless cubicle adjacent to the experimenter's room. The latter contained all of the stimulus-generating equipment, a slave Tektronix 608 display to allow monitoring of the subject's display, and an intercom to allow two-way communication with the subject. The subject's head rested in a padded chin- and foreheadrest, which also had padded temple screws, and which was situated $57 \mathrm{~cm}$ from the display such that $1 \mathrm{~cm}$ on the screen subtended $1^{\circ}$ of visual angle. All external cues to vertical were removed through the draping of the area between the headrest and the screen with black cloth to form a rough viewing tunnel; and a matt black aluminum mask mounted on the face of the display restricted the visible screen area to a $6.75^{\circ} \mathrm{cir}$ cular area.

The image generator was modified so that it was automated via the minicomputer and a custom-designed interface, which allowed up to three screens to be interleaved at a rate of $188 \mathrm{~Hz}$. A designer program allowed these separate screens to be constructed by a software-controlled menu.

Stimuli. The $0.6^{\circ}$ circular test grating had a spatial frequency of $5 \mathrm{cpd}$ and, when presented alone, was centered on a background luminance of $2.8 \mathrm{~cd} / \mathrm{m}^{2}$. The light and dark sinusoidal bars had respective luminances of 10.4 and $2.1 \mathrm{~cd} / \mathrm{m}^{2}$, measured on a lowfrequency grating by sweeping across the grating with a Tektronix $\mathrm{J} 161^{\circ}$ digital luminance probe. Thus, Michelson contrast, defined as $\left(L_{\max }-L_{\min }\right) /\left(L_{\max }+L_{\min }\right)$, was 0.67 . The inducing grating was confined to a $1^{\circ}$ thick annulus, outside diameter $2.6^{\circ}$, which was interleaved with the test grating on test trials to produce the percept of a single, nonflickering compound display. The inducing annulus also had a spatial frequency of 5 cpd and a contrast of 0.67 . Between trials, the entire screen was blanked at $6.8 \mathrm{~cd} / \mathrm{m}^{2}$, which sufficed to eradicate afterimages.

For both direct and indirect TIs, there were six exposure durations of the combined test and inducing fields (test conditions), and each of these was paired with a similar exposure duration of the test grating alone (pretest conditions). The durations chosen were a linear series: $5.32-\mathrm{msec}$ frame multiples of $2,4,6,8,10$, and 12 gave exposure durations of $10.6,21.2,31.9,42.6,53.2$, and $63.8 \mathrm{msec}$.

Procedure. Each subject was run under either the direct or the indirect $\mathrm{TI}$ conditions, using single staircases that were grouped and randomly interleaved. Thus, there were three blocks of trials: Block 1 contained staircases for the pretests and tests for the 10and 20-msec flashes; Block 2 contained them for the 30 - and 40-msec flashes; Block 3 contained them for the 50- and 60-msec flashes. For each subject, however, the ordering of Blocks 1-3 was random, and within each block, the four staircases (two test, two pretest) were randomly interleaved. There was a short, approximately 2 -min, rest between blocks, and the entire session lasted about $1 \mathrm{~h}$. For direct and indirect effects, the inducing gratings were always tilted $15^{\circ}$ and $75^{\circ}$, respectively, clockwise of vertical. All points of subjective vertical (PSV) to the left of true vertical were signed negative, and those to the right were signed positive, so that direct and indirect effects (test minus pretest) were signed positive and negative, respectively.

The starting position for any staircase was randomly chosen but was confined to $\pm 10^{\circ}$ frdm vertical. Step size initially was $2.12^{\circ}$. Each staircase was run for 10 reversals, and after the first two, step size was reduced to $1.06^{\circ}$. The PSV was estimated by means of averaging the peaks and valleys of the last six reversals.

The subjects were instructed to make an accurate judgment and to respond only after stimulus offset, by pressing one of the outer switches to indicate whether the test grating had appeared tilted left or right of vertical. A small, light fixation dot appeared $1 \mathrm{sec}$ prior to stimulus onset both to warn the subject of the impending flash and to maintain central fixation. After each response, there was a 500-msec interval before the next fixation-point onset.

Subjects. The subjects, volunteers from an introductory psychology course, received nominal course credit for participation. There were 15 subjects in Experiment 1 (direct effects) and 23 in Experiment 2 (indirect effects).

\section{Results}

The mean illusions are shown by the combined cross and circle symbols in Figure 1-solid symbols for direct and open symbols for indirect effects. The direct TI at

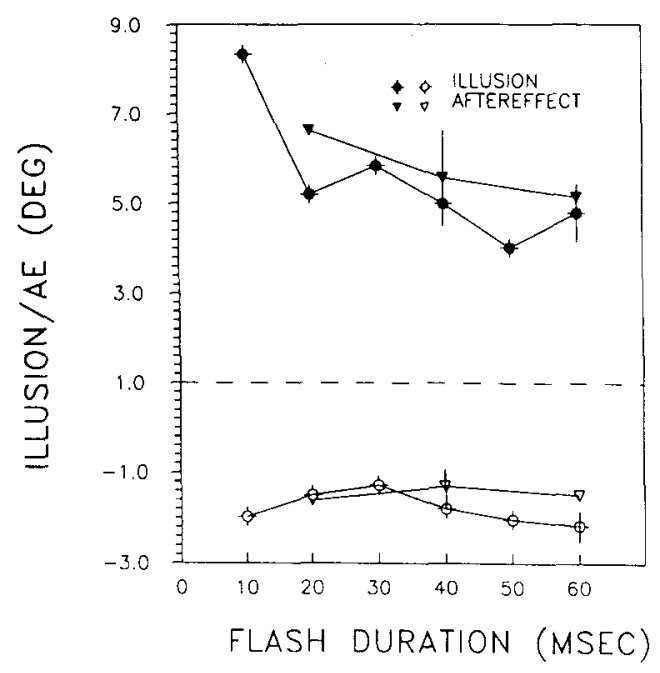

Figure 1. Direct (Experiment 1) and indirect TIs (Experiment 2) as a function of stimulus duration (combined circle and square symbols). Direct (Experiment 3) and indirect (Experiment 4) TAEs as a function of test-flash duration (inverted triangles). 
the 10-msec duration was $8.33^{\circ}$, consistent with Wolfe's (1984) TAE result in which four fifths of his subjects obtained TAEs greater than $5^{\circ}$ at that duration, effects too large to be measurable with his technique.

A simple treatments-by-subjects analysis with planned orthogonal contrasts (Winer, 1962, chap. 4) showed that direct TIs decreased with exposure duration both linearly $[F(1,70)=104.2 p<.0005]$ and quadratically $(F=$ $30.69, p<.0005)$. Together, these trends accounted for $81 \%$ of the treatments sum of squares. For indirect TIs, on the other hand, neither the linear nor the quadratic trend was significant, with $F(1,110)=1.19$ and 2.49 , respectively ( $p>.05$ in both cases). Nevertheless, the mean indirect TI over all exposures was $-1.80^{\circ}$, much larger than those usually obtained in experiments involving longduration stimuli. It is interesting that although indirect TI trends were nonsignificant, there appeared to be a slight dip in magnitude around $20-30 \mathrm{msec}$, as noted in previous data. Before discussing these data further, let us consider Experiments 3 and 4, which deal with TAEs with short test durations.

\section{EXPERIMENTS 3 AND 4}

Prior to Experiment 3 (direct TAE) and Experiment 4 (indirect TAE), pilot studies were conducted to ascertain task difficulty. Initially, we used the same display as in Experiments 1 and 2, except that the inducing stimulus was a complete $4.5^{\circ}$-diam circular grating rather than an annulus. One of us (P.W.) adapted for $2 \mathrm{~min}$ to a $+15^{\circ}$ grating, which was followed immediately by a short test flash. This in turn was followed by a 6-sec adapting " topup," another test flash, another "top up," and so on. Under these conditions, it proved impossible to see, let alone judge, the test flash. Accordingly, the task was made easier in three ways. First, the adapting stimuli and test flashes were always separated by a 500 -msec blank interval, as used by Wolfe (1984), although we used a light $\left(6.8 \mathrm{~cd} / \mathrm{m}^{2}\right)$ rather than a dark interval. Second, the diameter of the test stimulus was increased from $0.6^{\circ}$ to $1.0^{\circ}$. Third, relatively long test flashes, $20-60 \mathrm{msec}$, were used. We, and the volunteer subjects, were then able to do the task.

\section{Method}

Procedure. The subjects first adapted for $2 \mathrm{~min}$ to either a $+15^{\circ}$ (Experiment 3) or a $+75^{\circ}$ (Experiment 4) tilted grating and were instructed to move their eyes roughly circularly around the grating to avoid buildup of afterimages. However, to ensure ease of judgment, a small fixation dot was present at the center of the inducing grating, and $10 \mathrm{sec}$ before adaptation offset, the subjects were instructed to fixate it in readiness for the test flash. Test flashes were replaced with 6-sec "top-up" adaptation stimuli, during which central fixation was constant. Three test-flash durations were used: 21.2, 42.6, and $63.8 \mathrm{msec}$. Each of these adapting conditions was preceded by a pretest session in which only test flashes were presented, spaced by 10 -sec fixation. In both test and pretest conditions, single staircases began randomly $\pm 10^{\circ}$ from vertical and continued for 10 reversals, the last 6 of which were used to estimate the PSV. Between the three sets of adaptation sessions, the subjects remained in the dark for $5 \mathrm{~min}$, to allow dissipation of TAEs prior to the next condition.

Subjects. The subjects were from the same population as in previous experiments. Because it was clear at once that direct effects were large and comparable to those obtained with flashed TIs, only 5 subjects were run in Experiment 3. Fifteen subjects completed Experiment 4.

\section{Results}

The results are shown by the inverted triangles in Figure 1, and the TAE data clearly were comparable to those of the TI. Analysis of the direct effect data, as before, showed that the linear trend was significant $[F(1,8)=5.46, p<.05]$, accounting for $94 \%$ of the treatments sum of squares. In the case of indirect effects, neither the linear nor the quadratic trend was significant $[F(1,28)=0.1$ and 0.5 , respectively, $p>.05]$.

\section{Discussion}

Experiments 1-4 have provided some data relevant to Questions 1-3 above. First, Experiments 1 and 2 showed that even at the very shortest durations, direct TIs continue to increase, whereas indirect TIs, although they are large at shorter durations, show no consistent trend. Second, Experiments 3 and 4 have shown that short-duration TAEs are of magnitudes comparable to TIs, with direct TIs also showing an increase at shorter durations but with indirect TAEs exhibiting no consistent trend. To emphasize both the similarity of short-duration TIs and TAEs, and to show consistency with previous data, the results of Experiments 1-4 have been superimposed on those of Wenderoth and Johnstone (1988b) to produce Figure 2.

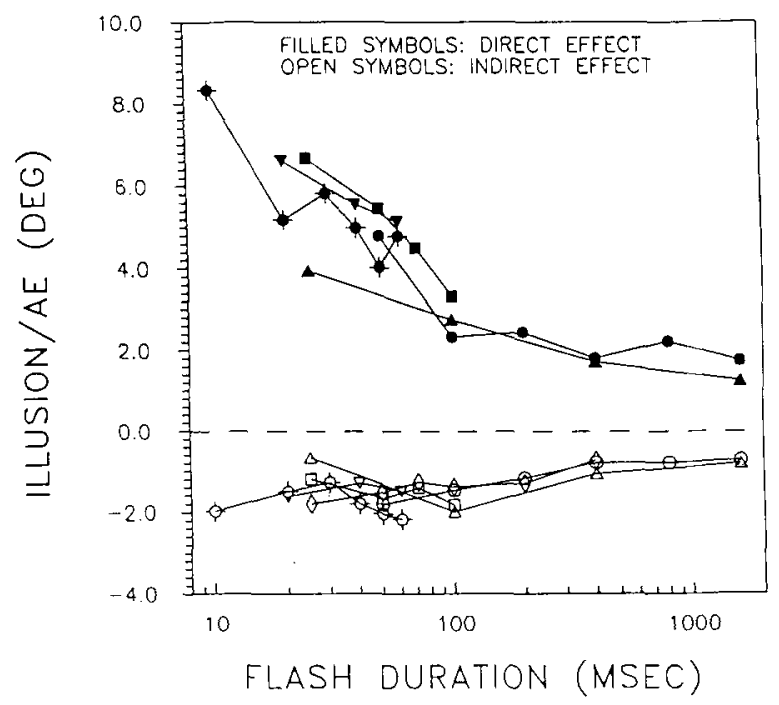

Figure 2. Data from Experiments 1-4 superimposed on those of Wenderoth and Johnstone (1988b; reprinted by permission). Symbols for Experiments 1-4 data are common to those in Figure 1. 
Further work is needed to clarify the precise nature of the function relating the indirect effect magnitude to exposure duration.

\section{EXPERIMENTS 5 AND 6}

The fact that TAEs similar to TIs occur at short durations allows the remaining questions to be addressed. First, since a surrounding square frame reduces indirect but not direct TIs, is the same true of TAEs, as would be expected if TIs and TAEs share common mechanisms? Second, does the frame, if it has this effect, influence the TAE at input (during adaptation) or output (during test)? Experiments 5 (direct TAE) and 6 (indirect TAE) were designed to address these questions.

\section{Method}

Procedure. All procedures and stimuli were identical to those in Experiments 3-4, but for the following exceptions. First, test ${ }^{\text {j }}$ flash duration was always $63.8 \mathrm{msec}$. Second, in some conditions, a square luminance frame surrounded either the adapting stimulus or the test stimulus. This frame was $5 \mathrm{~cm}$ on each side, internal luminance $3.0 \mathrm{~cd} / \mathrm{m}^{2}$ on a background $1.3 \mathrm{~cd} / \mathrm{m}^{2}$. Each subject completed four conditions: square in adapt only; square in test only; square in both; and square in neither.

Subjects. The subjects were drawn from the same population as before, with 21 in Experiment 5 and 29 in Experiment 6.

\section{Results}

The mean TAEs are shown in Figures 3 (direct TAEs) and 4 (indirect TAEs). Planned contrast analyses, as before, were used to test the main effects of the presence or absence of the frame during adaptation, of its presence or absence during the test, and the interaction. For direct effects, neither main effect was significant, with $F(1,60)=$ 0.99 and 0.02 , respectively $(p>.05)$. The interaction was significant $(F=5.05, p<.05)$. However, all four mean TAEs were clearly large and significant: there was no suggestion that the frame reduced TAEs in the manner found previously for indirect TIs. In the case of indirect TAEs, on the other hand, whereas the main effect of the

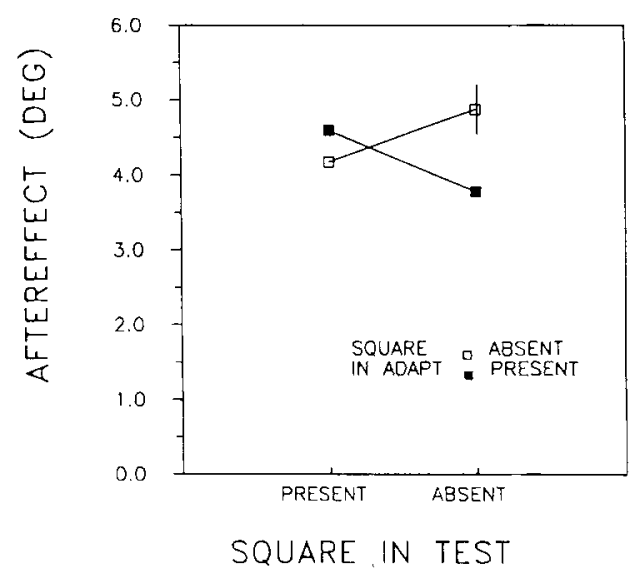

Figure 3. Direct TAE as a function of the presence or absence of surround frame in adapt and test conditions.

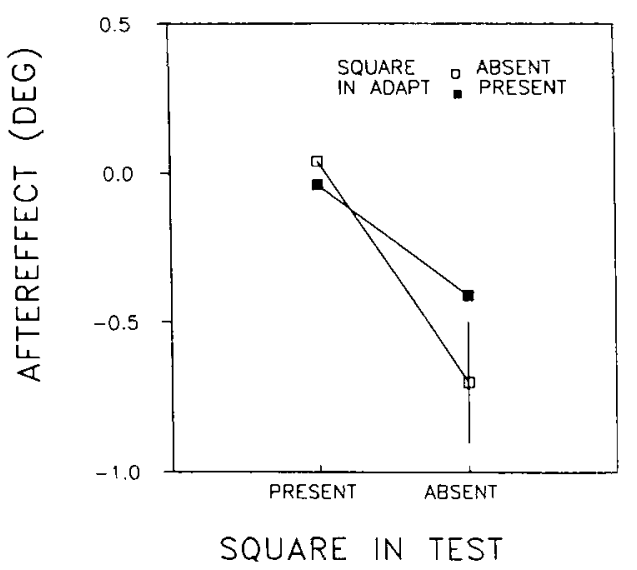

Figure 4. Indirect TAE as a function of the presence or absence of surround frame in adapt and test conditions.

presence or absence of the frame during adaptation was not significant $[F(1,84)=0.28, p>.05]$, the main effect during test was significant $(F=7.47, p<.01)$. The interaction was not significant $(F=0.79$ and $p>.05$ ). When the square was absent in the test, the overall mean TAE was $-0.55^{\circ}$. When it was present in the test, the TAE reduced to zero. There appeared to be some suggestion of an effect of the square in the adapt condition only, and we wondered whether the short fixation of the adapting stimulus might have resulted in the carryover of an afterimage of the frame to the test. It would be interesting to vary the interstimulus interval between adaptation and test conditions, to see whether the TAE increases with this interval, as it would if an afterimage of the square was present and decaying.

\section{GENERAL DISCUSSION}

The data from Experiments 5 and 6 show that the frame selectively reduces indirect, not direct, TAEs, just as it does in the case of the TI. The experiments also suggest strongly that the frame has its effect in the test, not during adaptation, so that it has a response effect rather than modifying the nature of neural adaptation at input. The fact that the remote frame contributes to the judgment of the test grating in the case of the indirect but not the direct effect may reflect the more global nature of receptive field mechanisms involved in the indirect effect. That the indirect effect may relate more to judgmental biases than to neural modification during adaptation is perhaps consistent with the view of Wenderoth and Johnstone (1988a), who attempted to relate indirect TIs to Allman et al.'s (1985) suggestion that TRFs may provide the basis for perceptual constancies. Thus, Wenderoth and Johnstone (1988a) speculated that

\footnotetext{
the indirect effect could be seen as arising from such global mechanisms involved in orientation constancy. Under normal circumstances, a rich collection of cues to vertical and horizontal ... is available in the visual field so that orien-
} 
tation coding errors are minimised. However, under impoverished conditions in the laboratory, when the inducing stimulus is the sole reference orientation, errors occur. This could explain why the surrounding frame ... removed indirect effects by providing the global orientation mechanisms with additional orientation data. (p. 310)

The fact that the frame in Experiment 6 had its effect mainly in the test phase rather than the adapting phase can be regarded as consistent with the constancy mechanism. On the other hand, that the direct TAE persists despite the frame's presence in the test suggests that the direct effect produces more local neural modification. When Köhler and Wallach (1944) first observed that indirect TAEs failed to occur when truly vertical edges were visible, they concluded: "In view of this difference between 'direct' and 'indirect' effects we hesitate to believe that the latter phenomenon is a figural after-effect in the sense in which we are using this term" (pp. 311-312). Our previous experiments had suggested different mechanisms for the direct and indirect TI. The experiments reported here reinforce that view, show that the same conclusion can be drawn regarding the direct and indirect TAE, but also reinforce Köhler and Wallach's claim that the indirect TAE (and presumably the TI) is not attributable to the same kind of local neural adaptation or inhibition as the direct TAE, because the latter effect cannot be overridden by remote cues to orientation presented in the test. In addition, the results of Experiments 3 and 4 have shown, once again, that indirect effects, unlike direct effects, are not systematically magnified by short-duration stimuli, suggesting, perhaps, that transient mechanisms underlie short-duration direct but not indirect effects.

\section{REFERENCES}

Allman, J. M., Miezin, F., \& McGuinness, E. (1985). Stimulus specific responses from beyond the classical receptive field: Neurophysiological mechanisms for local-global comparisons in visual neurons. Annual Review of Neuroscience, 8, 407-430.

Blakemore, C., Carpenter, R. H. S., \& Georgeson, M. A. (1970). Lateral inhibition between orientation detectors in the human visual system. Nature, 228, 37-39.

Calvert, J. E., Harris, J. P. (1988). Spatial frequency and duration effects on the tilt illusion and orientation acuity. Vision Research, 28, 1051-1059.

Carpenter, R. H. S., \& Blakemore, C. (1973). Interactions between orientations in human vision. Experimental Brain Research, 18, 287-303.

Desimone, R., Schein, S. J., Moran, J., \& Ungerleider, L. G. (1985). Contour, color and shape analysis beyond the striate cortex. Vision Research, 25, 441-452.

Georgeson, M. A. (1973). Spatial frequency selectivity of a visual tilt illusion. Nature, 245, 43-45.
Gibson, J. J., \& Radner, M. (1937). Adaptation, aftereffect and contrast in the perception of tilted lines: I. Quantitative studies. Journal of Experimental Psychology, 20, 453-467.

Harris, J. P., \& Calvert, J. E. (1989). Contrast, spatial frequency and test duration effects on the tilt aftereffect: Implications for underlying mechanisms. Vision Research, 29, 129-135.

KöHLER, W., \& WalLaCh, H. (1944). Figural-aftereffects: An investigation of visual processes. Proceedings of the American Philosophical Society, 88, 269-357.

LENNIE, P. (1972). Mechanisms underlying the perception of orientation. Unpublished doctoral dissertation, University of Cambridge.

Magnussen, S., Kurtenbach, W. (1980). Linear summation of tilt illusion and tilt aftereffect. Vision Research, 20, 39-42.

Maunsell, J. H. R., \& Newsome, W. T. (1987). Visual processing in monkey extrastriate cortex. Annual Review of Neuroscience, 10, 363-402.

Mitchell, D. E., Muir, D. W. (1976). Does the tilt aftereffect occur in the oblique meridian? Vision Research, 16, 609-613.

MoRANT, R. B., \& HARRIS, J. R. (1965). Two different aftereffects of exposure to visual tilts. American Journal of Psychology, 78, 218-226.

MuIR, D., OvER, R. (1970). Tilt aftereffects in central and peripheral vision. Joumal of Experimental Psychology, 85, 165-170.

OTOOLE, B. I., \& WENDEROTh, P. (1977). The tilt illusion: Repulsion and attraction effects in the oblique meridian. Vision Research, 17, 367-374.

Over, R., Broerse, J., \& Crassini, B. (1972). Orientation illusion and masking in central and peripheral vision. Joumal of Experimental Psychology, 96, 25-31.

Peterhans, E., von der Heydt, R. (1987). The whole and the pieces-cortical neuron responses to bars and rows of moving dots. In Seeing contour and colour. Satellite symposium conducted at the 2nd World Congress of Neuroscience, Manchester, England.

Tolnurst, D. J., \& Thompson, P. G. (1975). Orientation illusions and aftereffects: Inhibition between channels. Vision Research, 15, 967-972.

Virsu, V., TASKINEN, H. (1975). Central inhibitory interactions in human vision. Experimental Brain Research, 23, 65-74.

Wallace, G. K. (1969). The critical distance of interaction in the Zöllner illusion. Perception \& Psychophysics, 5, 261-264.

WARE, C., Mitchell, D. E. (1974). The spatial selectivity of the tilt aftereffect. Vision Research, 14, 735-737.

WENDEROTh, P., \& JoHNSON, M. (1985). What is the appropriate control for the tilt illusion? Perception, 14, 275-283.

Wenderoth, P., Johnstone, S. (1987). Possible neural substrates for orientation analysis and perception. Perception, 16, 693-709.

Wenderoth, P., \& Johnstone, S. (1988a). The different mechanisms of the direct and indirect tilt illusions. Vision Research, 28, 301-312.

Wenderoth, P., Johnstone, S. (1988b). The differential effects of brief exposures and surrounding contours on direct and indirect tilt illusions. Perception, 17, 165-176.

Wenderoth, P., O'ConNOR, T., \&ohnson, M. (1986). The tilt illusion as a function of the relative and absolute lengths of test and inducing lines. Perception \& Psychophysics, 39, 339-345.

WINER, B. J. (1962). Statistical principles in experimental design. New York: McGraw-Hill.

WoLFE, J. M. (1984). Short test flashes produce large tilt aftereffects. Vision Research, 24, 1959-1964.

(Manuscript received October 19, 1988; revision accepted for publication March 20, 1989.) 